\title{
Assessing the Need for Adjuvant Chemotherapy After Stereotactic Body Radiation Therapy in Early-stage Non-small Cell Lung Carcinoma
}

Raphaël Jumeau ${ }^{1}$, Houda Bahig ${ }^{2}$, Édith Filion ${ }^{2}$, Marie-Pierre Campeau ${ }^{1}$, Louise Lambert ${ }^{2}$, David Roberge $^{3}$, Andrei-Bogdan Gorgos ${ }^{4}$, Toni $\mathrm{Vu}^{2}$

1. Department of Radiation Oncology, Centre Hospitalier de l'Université de Montréal (CHUM) 2. Department of Radiation Oncology, Centre hospitalier de l'Université de Montréal (CHUM) 3. Radiation Oncology, University of Montreal Health Centre, Montréal, CAN 4. Radiology, Centre hospitalier de l'Université de Montréal (CHUM)

$\square$ Corresponding author: Raphaël Jumeau, raphael.jumeau@gmail.com Disclosures can be found in Additional Information at the end of the article

\section{Abstract \\ Purpose}

Surgery remains the standard treatment for medically operable patients with early-stage nonsmall cell lung carcinoma (NSCLC). Following surgical resection, adjuvant chemotherapy is recommended for large tumors $>4 \mathrm{~cm}$. For unfit patients, stereotactic body radiation therapy (SBRT) has emerged as an excellent alternative to surgery. This study aims to assess patterns of recurrence and discuss the role of chemotherapy after SBRT for NSCLC.

\section{Methods}

We reviewed patients treated with SBRT for primary early-stage NSCLC between 2009 and 2015. Total target doses were between 50 and $60 \mathrm{~Gy}$ administered in three to eight fractions. All patients had a staging fluorodeoxyglucose (FDG) positron emission tomography (PET) integrated with computed tomography (CT) scan, and histologic confirmation was obtained whenever possible. Mediastinal staging was performed if lymph node involvement was suspected on CT or PET/CT. Survival outcomes were estimated using the Kaplan-Meier method.

\section{Results}

Among the 559 early-stage NSCLC patients treated with SBRT, 121 patients were stage T2N0. The one-year and three-year overall survival rates were $88 \%$ and $70 \%$, respectively, for patients

Received 09/21/2016 Review began 09/28/2016 Review ended 11/14/2016 Published 11/29/2016

\section{(c) Copyright 2016}

Jumeau et al. This is an open access article distributed under the terms of the Creative Commons Attribution License CC-BY 3.0., which permits unrestricted use, distribution, and reproduction in any medium, provided the original author and source are credited. with $\mathrm{T} 2$ disease, compared to $95 \%$ and $81 \%$, respectively, for the T1 patients $(\mathrm{p}<0.05)$. The oneyear and three-year local control rates were equal in both groups (98\% and 91\%, respectively). In T2 patients, 25 (21\%) presented a relapse, among which 21 (84\%) were nodal or distant. The median survival of T2N0 patients following a relapse was 11 months.

\section{Conclusion}

Lung SBRT provides high local control rates, even for larger tumors. When patients relapse, the majority of them do so at regional or distant sites. These results raise the question as to whether adjuvant treatment should be considered following SBRT for larger tumors.

\section{How to cite this article}

Jumeau R, Bahig H, Filion É, et al. (November 29, 2016) Assessing the Need for Adjuvant Chemotherapy After Stereotactic Body Radiation Therapy in Early-stage Non-small Cell Lung Carcinoma. Cureus 8(11): e901. DOI 10.7759/cureus.901 
Categories: Radiation Oncology, Oncology

Keywords: lung cancer, sbrt, adjuvant chemotherapy

\section{Introduction}

Lung cancer is the most common cause of death from cancer worldwide [1]. Among all histological types, NSCLC is the most frequent [2]. Although SBRT is being prospectively compared to surgical resection for medically operable patients, the standard management of early-stage NSCLC remains surgical lobectomy [3].

The role of adjuvant treatment after surgery has been studied extensively. In 1995 the NSCLC collaborative group [4] published the first meta-analysis supporting the use of adjuvant chemotherapy. More recently, a larger meta-analysis [5] based on 4,584 patients suggested that adjuvant cisplatin-based chemotherapy significantly improves survival. Current guidelines recommend adding chemotherapy after complete resection for patients with high-risk tumors: vascular invasion, wedge resection, visceral pleural involvement, unknown lymph node status and tumors $>4 \mathrm{~cm}[6]$.

For patients who are medically unfit or who decline surgery, SBRT has emerged as the favored alternative. It provides local control rates comparable to surgery with low toxicity [7]. However, adjuvant treatment is rarely considered after lung SBRT, even for those with larger tumors. In this study, we present our results for patients treated with SBRT for high-risk early-stage NSCLC and discuss the potential benefit of adjuvant treatment.

\section{Materials And Methods}

\section{Patients and tumors}

We retrospectively reviewed patients treated with SBRT for NSCLC at our institution between July 2009 and August 2015. Pretreatment workup included a diagnostic CT, PET/CT, bronchoscopy and pulmonary function testing with measurements of forced expiratory volume in one second (FEV1) and diffusing capacity of the lung for carbon monoxide (DLCO) [8].

Mediastinal staging (MS) was performed if lymph node involvement was suspected on CT or PET/CT. Histological confirmation was sought by bronchoscopy or transthoracic needle biopsy. When appropriate, gold fiducials were placed during percutaneous lung biopsy to allow tumor tracking. If the biopsy was impossible or inconclusive, radiological and clinical criteria were followed [9]. Measurements of the lesions were based on the largest dimension in axial view on the diagnostic CT. We defined central lesions as tumors within $2 \mathrm{~cm}$ of the proximal tracheobronchial tree or within $2 \mathrm{~cm}$ of other mediastinal structures [10].

\section{Treatment planning and delivery}

SBRT was delivered using a variety of radiotherapy platforms: helical tomotherapy, CyberKnife ${ }^{\circledR}$ robotic radiotherapy (Accuray Inc., Sunnyvale, CA, USA) or isocentric linear accelerators with volumetric modulated arc therapy (VMAT). Dose schedules were 60 Gy in three to five fractions for peripheral lesions, and $50 \mathrm{~Gy}$ in five fractions or $60 \mathrm{~Gy}$ in eight fractions for central lesions.

Patients were treated either with near-real-time tumor tracking with CyberKnife ${ }^{\circledR}$ or using an internal target volume (ITV) with VMAT or helical tomotherapy. CyberKnife ${ }^{\circledR}$ tumor tracking was achieved using fiducials or, when tumor was sufficiently large and dense, using a soft tissue tracking technique (Xsight Lung, Accuray Inc., Sunnyvale, CA, USA) [11]. 
All patients had a noncontrast 4D planning CT scan in supine position. The gross tumor volume (GTV) was delineated on the expiratory phase of 4D CT and corresponded to the macroscopic tumor on pulmonary CT windows. For patients treated with VMAT or helical tomotherapy, a BodyFIX (Elekta, Stockholm, Sweden) whole body vaccum immobilization device was also used. ITV was based on tumor motion in extreme phases of the respiratory cycle. An additional planning tumor volume (PTV) margin of $5 \mathrm{~mm}$ was added to the GTV in fiducials or Xsight Lung cases, or to the ITV, alternatively.

\section{Follow-up and endpoints}

After completion of SBRT, patients were first seen at three months for a detailed history and physical exam, then every six months thereafter. A chest CT scan was performed before each visit.

The primary endpoint was the local control (LC) evaluated by radiologists based on follow-up CT scans. LC was defined radiologically by the absence of a growing lesion within the involved lobe on sequential follow-up CT scans [12]. If lymph node involvement or metastatic evolution was suspected during follow-up, patients underwent whole-body PET/CT to confirm the nonlocal (nodal or distant) relapse. A histological confirmation of the relapse was performed when possible.

\section{Statistics}

Statistical analysis was performed by SPSS software (SPSS Inc., Chicago, IL, USA). The local control, nodal control, distant control and survival outcomes were estimated using the KaplanMeier method. Data were collected and analyzed using Student's t-test, Chi square and Fisher's exact test. $P$ values less than 0.05 were considered statistically significant.

IRB approval, number 09.029, was provided by Comité d'éthique de la recherche du CHUM. Patient informed consent was obtained.

\section{Results}

\section{Population and tumor characteristics}

Between July 2009 and August 2015, 574 treatments had been delivered in 559 patients. Patient and lesion characteristics are summarized in Table 1. 


\section{Cureus}

\begin{tabular}{|c|c|c|c|}
\hline & T1 & T2 & $P$ value \\
\hline Patients & 438 & 121 & \\
\hline Age (years) & 73 & 76 & $<0.05$ \\
\hline KPS & 90 & 80 & $>0.1$ \\
\hline FEV1 (L) & 1.2 & 1.3 & $>0.1$ \\
\hline FEV1 (\%) & 62 & 66 & $>0.1$ \\
\hline DLCO (\%) & 57 & 57 & $>0.1$ \\
\hline Lesions & 451 & 123 & \\
\hline Size (cc) & 1.8 & 3.6 & $<0.05$ \\
\hline Central/peripheral & $77 / 374$ & $52 / 71$ & $<0.05$ \\
\hline Histology (\%) & 71 & 86 & $<0.05$ \\
\hline Adenocarcinoma & 36 & 39 & \\
\hline Squamous cell carcinoma & 20 & 31 & \\
\hline Large cell carcinoma & 2 & 2 & \\
\hline Other specified carcinoma & 6 & 9 & \\
\hline Mediastinal staging (\%) & 14 & 21 & $<0.05$ \\
\hline
\end{tabular}

\section{TABLE 1: Patients and tumors characteristics}

KPS: Karnofsky Performance Scale; FEV1: forced expiratory volume in one second; DLCO: diffusing capacity of the lung for carbon monoxide

\section{Cohort outcomes}

The median follow-up time was 16 months (range: one to 62). LC rates were equal in the T1 and T2 groups: $98 \%$ and $91 \%$ at one and three years, respectively. Non-local control rates were different between T1 and T2 patients: $93 \%$ vs. $89 \%$ at one year and $83 \%$ vs. $69 \%$ at three years ( $\mathrm{p}<0.05)$, respectively. Overall survival (OS) at one and three years was also statistically different between the two groups: $95 \%$ and $81 \%$ in $\mathrm{T} 1$ patients and $88 \%$ and $70 \%$ in $\mathrm{T} 2$ patients $(\mathrm{p}<0.05)$, respectively (Figure 1$)$. The percentage of deaths attributable to lung cancer was $42 \%$ in the $\mathrm{T} 1$ group, while it was $52 \%$ in the T2 group ( $\mathrm{p}>0.1$ ) (Table 2). Disease-free survival (DFS) was $88 \%$ at one year and $66 \%$ at three years in the T1 group, and it was $79 \%$ and $51 \%$ in the T2 group $(\mathrm{p}<0.05)$, respectively (Figure 2$)$. 


\section{Cureus}

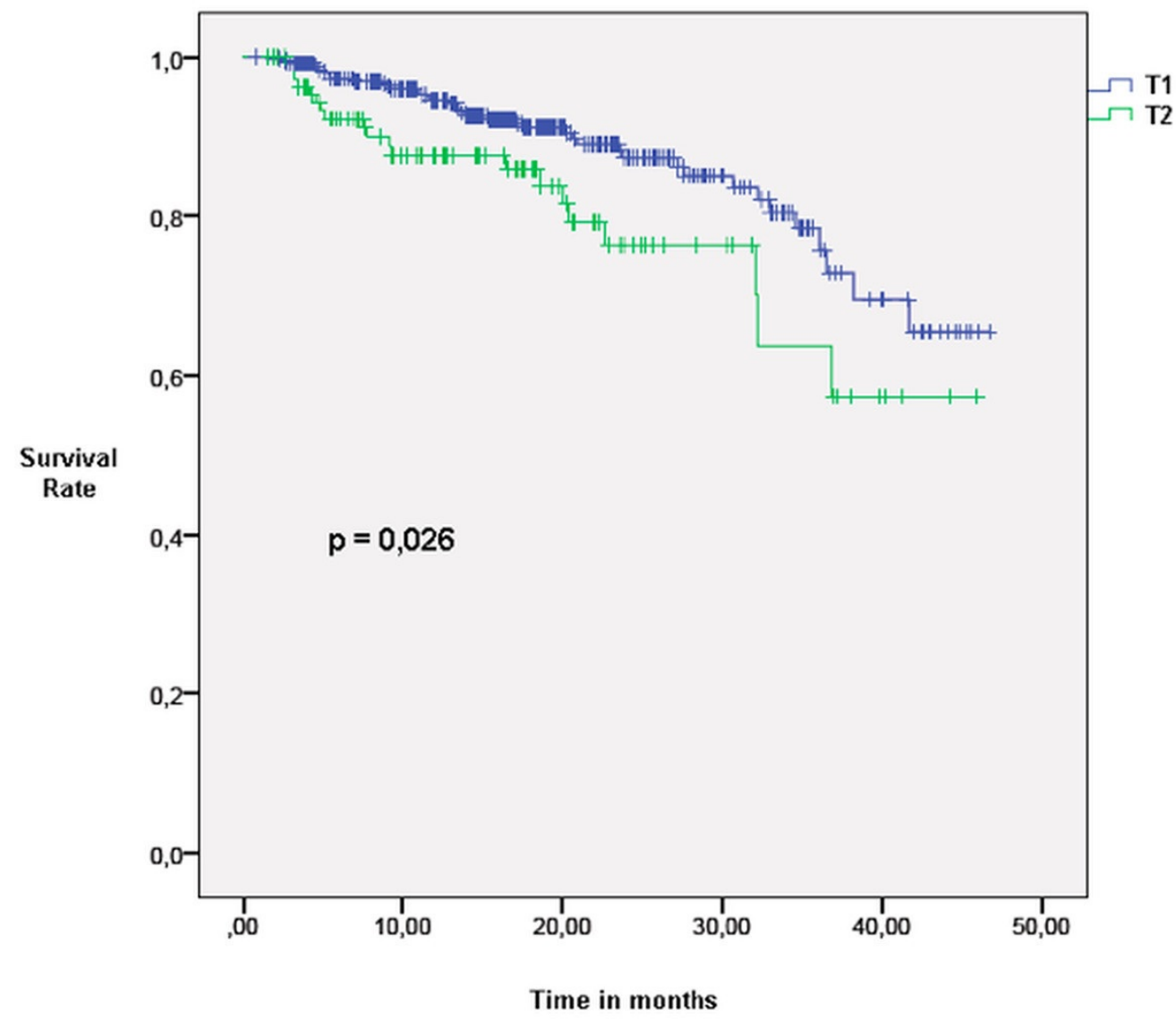

FIGURE 1: Overall survival in T1 and T2 patients

\begin{tabular}{|c|c|c|c|}
\hline & T1 & T2 & $P$ value \\
\hline Total number of deaths & 43 & 21 & \\
\hline Number of lung cancer deaths & 18 & 11 & \\
\hline$\%$ & 42 & 52 & $>0.1$ \\
\hline RR & \multicolumn{2}{|c|}{1.25} & \\
\hline $95 \% \mathrm{Cl}$ & \multicolumn{2}{|c|}{$[0.5 ; 4.3]$} & \\
\hline
\end{tabular}

\section{TABLE 2: Death statistics}

RR: relative risk

$\mathrm{Cl}$ : confidence interval 


\section{Cureus}

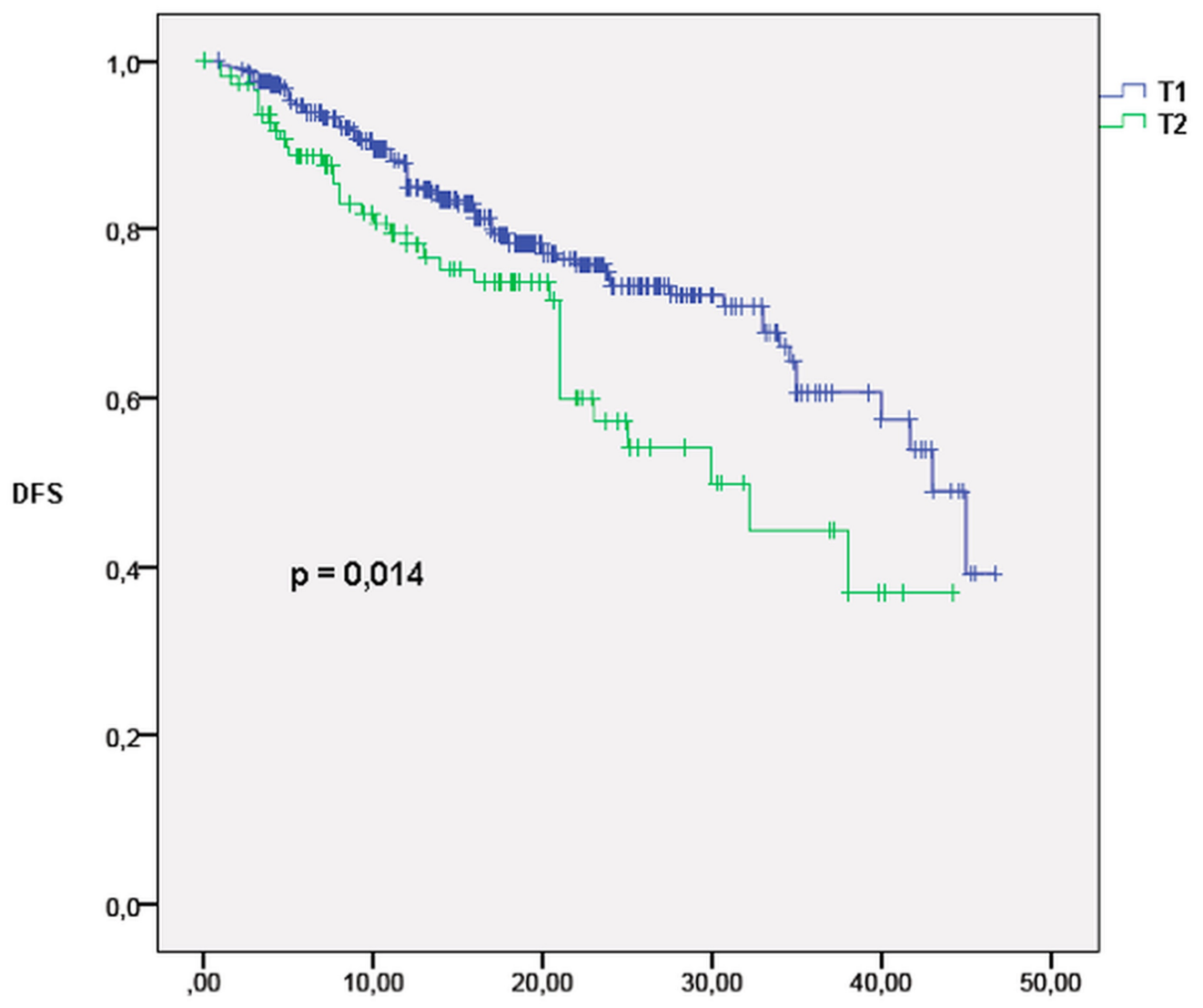

Time in months

FIGURE 2: Disease-free survival in T1 and T2 patients

\section{T2 patients}

Among the $121 \mathrm{~T} 2$ patients, six were staged T2b. Unsurprisingly, survival of T2 patients without relapse was higher than T2 patients having suffered a relapse $(\mathrm{p}<0.05)$ (Figure 3). The median survival of T2N0 patients following a relapse was 11 months. Twenty-five patients (21\%) presented a relapse: four (16\%) were local and 21 (84\%) were nodal or distant. Among the nonlocal relapses, eight (38\%) were nodal and 13 (62\%) were distant. Two patients with distant relapse limited to the brain had local treatment (one radiosurgery and one surgery). In the rest of the non-local relapse group, six patients (32\%) were considered eligible to receive palliative chemotherapy. One patient (5\%) declined chemotherapy and three (16\%) received cytotoxics, while two (10\%) had targeted therapy. 


\section{Cureus}

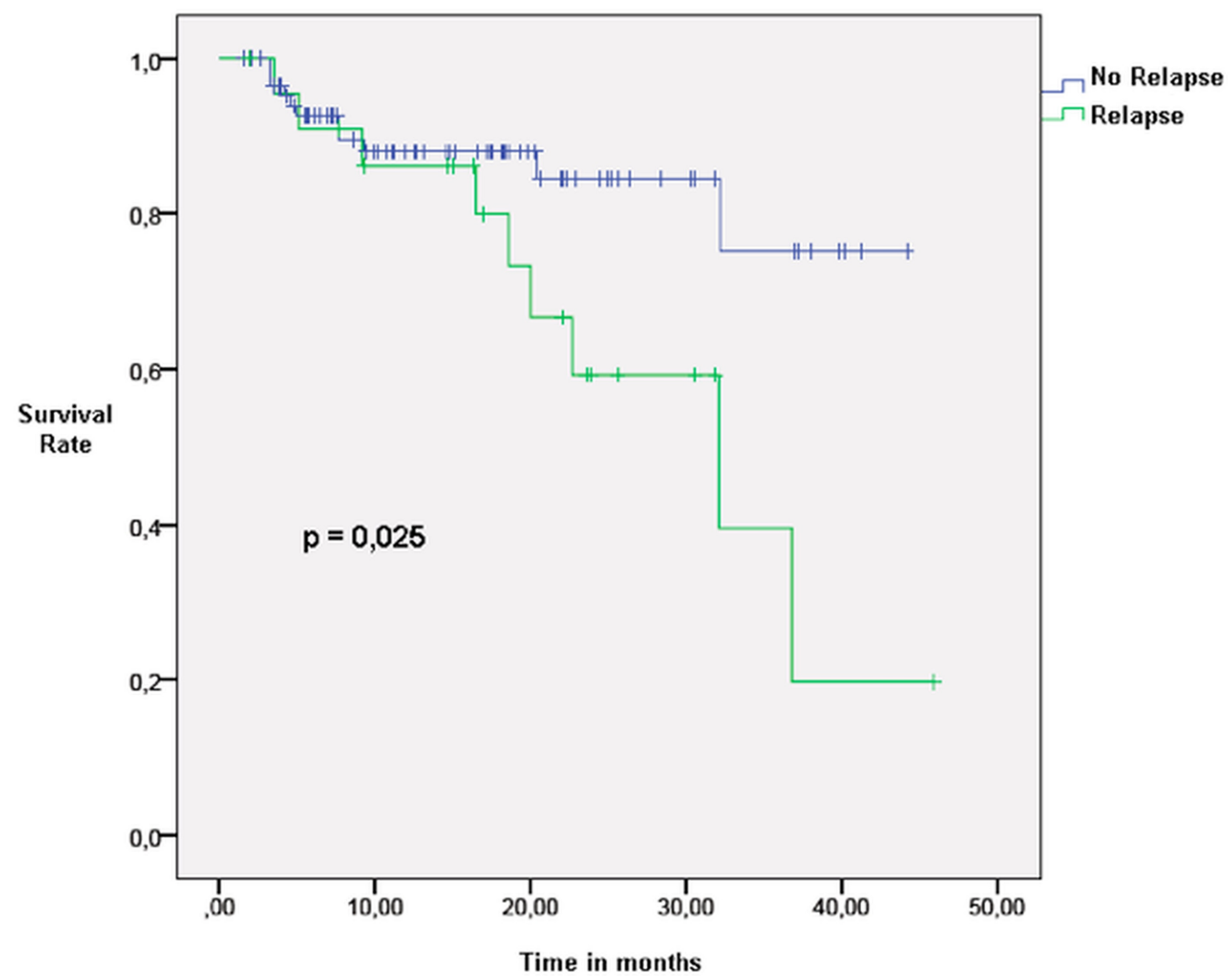

FIGURE 3: Overall survival in T2 patients after the diagnosis of relapse

\section{Discussion}

Adjuvant chemotherapy is a well-established treatment after complete surgical resection of NSCLC tumors $>4 \mathrm{~cm}$. For nonsurgical patients, SBRT is now considered standard treatment. Even if SBRT is a highly efficient local treatment, there is a lack of data available concerning the possibility of adjuvant treatment after lung SBRT for large NSCLC tumors.

In our study, we confirmed that SBRT provides excellent LC with a three-year rate of 91\%. This result is comparable to rates previously reported for NSCLC SBRT [13-19]. Furthermore, LC is similar between the $\mathrm{T} 1$ and $\mathrm{T} 2$ groups and comparable to that reported in surgical series. In fact, Kastelijn et al. [20], in a retrospective study, compared clinical outcomes of SBRT ( $N=53$ ) versus surgical resection $(\mathrm{N}=175)$ for stage I-II NSCLC. They showed similar results in terms of OS and progression-free survival between surgery and SBRT. Chang et al. [21], in a pooled analysis of two randomized trials comparing surgery and SBRT for operable stage I (T1a, T1b and T2a) NSCLC, found similar results between the two treatments in terms of LC and DFS; the three-year OS was higher for SBRT patients ( $95 \%$ vs. $79 \%, \mathrm{p}=0.037$ ), but the number of patients was not sufficient to conclude $(\mathrm{N}=58)$.

In our study, non-local recurrence rates were also comparable to surgical series. Robinson et al. [22] studied patterns of failure after surgery (lobectomy or pneumonectomy) or SBRT for stage I NSCLC. In their retrospective study, they concluded to an advantage in OS for surgery, but the primary tumor LC rate $(98.7 \%$ vs. $95.3 \%, \mathrm{p}=0.088)$, the regional control rate $(82.9 \%$ vs. $78.1 \%$, $\mathrm{p}=0.912)$ and the distant control rate ( $76 \%$ vs. $54 \%, \mathrm{p}=0.152)$ were respectively similar for surgery versus SBRT. Moreover, in our study, we showed a clear majority of non-local 
recurrences for T2 patients. Comparable LC rates of lung SBRT with those of surgery, as well as the important rates of non-local recurrences, raise the question as to the benefit of an adjuvant treatment.

In a systematic review of the literature, Burdett et al. [23] showed that there is a clear advantage to add chemotherapy after surgery. Their analyses based on 8,447 patients over 34 trials proved an absolute increase of $4 \%$ in survival at five years. It must be pointed out that patients in these studies were younger (median age of 60 years) than lung cancer patients seen in clinical routine, including our practice. In an analysis of randomized trials by MadroszykFlandrin et al. [24], based on 71 patients with stage I-IV NSCLC, they showed that chemotherapy is feasible in elderly patients ( $\geqslant 70$ years). In fact, elderly patients could receive adjuvant chemotherapy but had more modest survival outcomes than younger patients. As analyzed by Früh et al. [25], based on patient data from the Lung Adjuvant Cisplatin Analysis (LACE), elderly patients had worse outcomes. They reported a higher rate of noncancerousrelated death, and elderly patients received lower doses of chemotherapy. Adding chemotherapy after SBRT seems possible, even for older patients, but a rigorous screening is necessary to select patients fit enough to receive the treatment.

In recent years, targeted therapies have emerged in NSCLC management. These treatments are considered safer because patients have less side effects. However, the role of targeted therapies after surgery is not clear [26]. The most represented targeted agents are the tyrosine kinase inhibitors (TKI) of epidermal growth factor receptor (EGFR). The BR-19 trial [27] enrolled 503 patients in a phase III study comparing surgery alone and surgery followed by EGFR-TKI (gefitinib) administration, regardless of the EGFR status. There was no difference in OS or DFS between the two groups. More recently, the RADIANT trial [28] studied adjuvant EGFR-TKI (erlotinib) treatment after resection of stage IB-IIIA NSCLC only for tumors with the expression of EGFR. In the subgroup of patients with EGFR mutation, DFS favored erlotinib (46.4 vs. 28.5 months), but this was not statistically significant. Regarding these results, targeted agents may provide a benefit as adjuvant treatment and have the advantage to be better tolerated than cytostatic drugs. It must be pointed out that they seem not to be indicated for patients without specific mutations.

One retrospective study of 65 patients by Chen et al. [29] suggested a benefit for adjuvant chemotherapy in T1-3N0 NSCLC following SBRT. The three- and five-year OS rates in the adjuvant chemotherapy group were $81 \%$ and $46 \%$, respectively, while they were $50 \%$ and $32 \%$, respectively, for patients who had not received chemotherapy. Patients who received adjuvant chemotherapy had a lower relapse rate. But the number of patients who received adjuvant chemotherapy $(\mathrm{N}=17)$ was not sufficient to provide significant results.

In our study, only one third of $\mathrm{T} 2$ patients were eligible for systemic treatment after the diagnosis of relapse. The majority of these patients (68\%) were unfit to receive systemic treatment and were directed to supportive care units. Although this may be an argument in favor of adjuvant treatment (when the patient may be more fit), it also highlights that a number of these patients may not be fit for chemotherapy after SBRT. OS and DFS are significantly worst in T2 patients. The shortness of the median survival time after diagnosis of relapse in the T2 group highlights the aggressiveness of the recurrence that is congruent with the median survival of 10-11 months described by Schiller et al. [30] in metastatic NSCLC receiving chemotherapy.

Adjuvant treatment for early-stage NSCLC after lung SBRT could potentially benefit selected patients after lung SBRT. However, SBRT patients tend to be older and frailer than patients eligible for surgery; therefore, fewer of them would be eligible for an adjuvant treatment. On the other hand, few patients received SBRT because they refused surgery, and they will probably refuse adjuvant treatment even if they are eligible for it. Nonetheless, our results showed that 
patients with larger tumors have a worse outcome, so further prospective studies with bigger cohorts are warranted.

\section{Conclusions}

Lung SBRT provides excellent local control rates, even for larger tumors. Overall survival and patterns of failure are similar to surgery. However, it remains unclear whether SBRT patients will be sufficiently fit for adjuvant chemotherapy, but these results show the need for future trials to study this question.

\section{Additional Information}

\section{Disclosures}

Human subjects: Consent was obtained by all participants in this study. Comité d'éthique de la recherche du CHUM issued approval 09.029. Animal subjects: All authors have confirmed that this study did not involve animal subjects or tissue. Conflicts of interest: In compliance with the ICMJE uniform disclosure form, all authors declare the following: Payment/services info: All authors have declared that no financial support was received from any organization for the submitted work. Financial relationships: David Roberge declare(s) a grant and personal fees from Varian Medical Systems. David Roberge declare(s) personal fees and non-financial support from Accuray. Other relationships: All authors have declared that there are no other relationships or activities that could appear to have influenced the submitted work.

\section{References}

1. Ferlay J, Soerjomataram I, Dikshit R, et al.: Cancer incidence and mortality worldwide: sources, methods and major patterns in GLOBOCAN 2012. Int J Cancer. 2015, 136:359-386. 10.1002/ijc. 29210

2. Herbst RS, Heymach JV, Lippman SM: Lung cancer. N Engl J Med. 2008, 359:1367-80. 10.1056/NEJMra0802714

3. Vansteenkiste J, Crinò L, Dooms C, et al.: 2nd ESMO Consensus Conference on Lung Cancer: early-stage non-small-cell lung cancer consensus on diagnosis, treatment and follow-up. Ann Oncol. 2014, 25:1462-74. 10.1093/annonc/mdu089

4. Alberti W, Anderson G, Bartolucci A, et al.: Chemotherapy in non-small cell lung cancer: a meta-analysis using updated data on individual patients from 52 randomised clinical trials. Non-small Cell Lung Cancer Collaborative Group. BMJ. 1995, 311:899-909. 10.1136/bmj.311.7010.899

5. Pignon JP, Tribodet H, Scagliotti GV, et al.: Lung adjuvant cisplatin evaluation: a pooled analysis by the LACE Collaborative Group. J Clin Oncol. 2008, 26:3552-9. 10.1200/JCO.2007.13.9030

6. Ettinger DS, Akerley W, Bepler G, et al.: Non-small cell lung cancer. J Natl Compr Canc Netw. 2010, 8:740-801.

7. Antoni D, Srour I, Noël G, Mornex F: Radiothérapie en conditions stéréotaxiques des tumeurs bronchopulmonaires [Article in French, English]. Cancer Radiother. 2014, 18:297-307. 10.1016/j.canrad.2014.05.002

8. Brunelli A, Charloux A, Bolliger CT, et al.: ERS/ESTS clinical guidelines on fitness for radical therapy in lung cancer patients (surgery and chemo-radiotherapy). Eur Respir J. 2009, 34:1741. 10.1183/09031936.00184308

9. Gould MK, Fletcher J, Iannettoni MD, et al.: Evaluation of patients with pulmonary nodules: when is it lung cancer?: ACCP evidence-based clinical practice guidelines (2nd edition). Chest. 2007, 132:108-30. 10.1378/chest.07-1353

10. Chang JY, Balter PA, Dong L, et al.: Stereotactic body radiation therapy in centrally and superiorly located stage I or isolated recurrent non-small-cell lung cancer. Int J Radiat Oncol Biol Phys. 2008, 72:967-71. 10.1016/j.ijrobp.2008.08.001

11. Bahig H, Campeau MP, Vu T, et al.: Predictive parameters of CyberKnife fiducial-less (XSight Lung) applicability for treatment of early non-small cell lung cancer: a single-center 
experience. Int J Radiat Oncol Biol Phys. 2013, 87:583-9. 10.1016/j.ijrobp.2013.06.2048

12. Huang K, Senthi S, Palma DA, et al.: High-risk CT features for detection of local recurrence after stereotactic ablative radiotherapy for lung cancer. Radiother Oncol. 2013, 109:51-7. 10.1016/j.radonc.2013.06.047

13. Aoki M, Hatayama Y, Kawaguchi H, et al.: Clinical outcome of stereotactic body radiotherapy for primary and oligometastatic lung tumors: a single institutional study with almost uniform dose with different five treatment schedules. Radiat Oncol. 2016, 11:5. 10.1186/s13014-0160581-2

14. Fukumoto SI, Shirato H, Shimzu S, et al.: Small-volume image-guided radiotherapy using hypofractionated, coplanar, and noncoplanar multiple fields for patients with inoperable Stage I nonsmall cell lung carcinomas. Cancer. 2002, 95:1546-53. 10.1002/cncr.10853

15. Timmerman R, Papiez L, McGarry R, et al.: Extracranial stereotactic radioablation: results of a phase I study in medically inoperable stage I non-small cell lung cancer. Chest. 2003, 124:1946-55. 10.1378/chest.124.5.1946

16. Onimaru R, Shirato H, Shimizu S, et al.: Tolerance of organs at risk in small-volume, hypofractionated, image-guided radiotherapy for primary and metastatic lung cancers. Int J Radiat Oncol Biol Phys. 2003, 56:126-35. 10.1016/S0360-3016(03)00095-6

17. Hof H, Herfarth KK, Münter M, et al.: Stereotactic single-dose radiotherapy of stage I nonsmall-cell lung cancer (NSCLC). Int J Radiat Oncol Biol Phys. 2003, 56:335-41.

18. Zimmermann FB, Geinitz H, Schill S, et al.: Stereotactic hypofractionated radiation therapy for stage I non-small cell lung cancer. Lung Cancer. 2005, 48:107-14.

10.1016/j.lungcan.2004.10.015

19. Koto M, Takai Y, Ogawa Y, et al.: A phase II study on stereotactic body radiotherapy for stage I non-small cell lung cancer. Radiother Oncol. 2007, 85:429-34. 10.1016/j.radonc.2007.10.017

20. Kastelijn EA, El Sharouni SY, Hofman FN, et al.: Clinical outcomes in early-stage NSCLC treated with stereotactic body radiotherapy versus surgical resection. Anticancer Res. 2015, 35:5607-14.

21. Chang JY, Senan S, Paul MA, et al.: Stereotactic ablative radiotherapy versus lobectomy for operable stage I non-small-cell lung cancer: a pooled analysis of two randomised trials. Lancet. 2015, 16:630-37. 10.1016/S1470-2045(15)70168-3

22. Robinson CG, DeWees TA, El Naqa IM, et al.: Patterns of failure after stereotactic body radiation therapy or lobar resection for clinical stage I non-small-cell lung cancer. J Thorac Oncol. 2013, 8:192-201. 10.1097/JTO.0b013e31827ce361

23. Burdett S, Pignon JP, Tierney J, et al.: Adjuvant chemotherapy for resected early-stage nonsmall cell lung cancer. Cochrane Database Syst Rev. 2015, 3:1-73.

10.1002/14651858.CD011430

24. Madroszyk-Flandin A, Bagattini S, Gonçalves A, et al.: Lung cancer in elderly patients: a retrospective analysis of practice in a single institution. Crit Rev Oncol Hematol. 2007, 64:438. 10.1016/j.critrevonc.2007.06.009

25. Früh M, Rolland E, Pignon JP, et al.: Pooled analysis of the effect of age on adjuvant cisplatinbased chemotherapy for completely resected non-small-cell lung cancer. J Clin Oncol. 2008, 26:3573-81. 10.1200/JCO.2008.16.2727

26. Tanaka F, Yoneda K: Adjuvant therapy following surgery in non-small cell lung cancer (NSCLC). Surg Today. 2016, 46:25-37. 10.1007/s00595-015-1174-7

27. Goss GD, O’Callaghan C, Lorimer I, et al.: Gefitinib versus placebo in completely resected non-small-cell lung cancer: results of the NCIC CTG BR19 study. J Clin Oncol. 2013, 31:33206. 10.1200/JCO.2013.51.1816

28. Kelly K, Altorki NK, Eberhardt WE, et al.: Adjuvant erlotinib versus placebo in patients with stage IB-IIIA non-small-cell lung cancer (RADIANT): a randomized, double-blind, phase III trial. J Clin Oncol. 2015, 33:4007-14. 10.1200/JCO.2015.61.8918

29. Chen Y, Guo W, Lu Y, Zou B: Dose-individualized stereotactic body radiotherapy for T1-3N0 non-small cell lung cancer: long-term results and efficacy of adjuvant chemotherapy. Radiother Oncol. 2008, 88:351-8. 10.1016/j.radonc.2008.07.013

30. Schiller JH, Harrington D, Belani CP, et al.: Comparison of four chemotherapy regimens for advanced non-small-cell lung cancer. N Engl J Med. 2002, 346:92-8. 10.1056/NEJMoa011954 